\title{
Programmed cell death-ligand 1 assessment in urothelial carcinoma: prospect and limitation
}

\author{
Kyu Sang Lee ${ }^{1,2}$, Gheeyoung Choe $\mathrm{e}^{1,2}$ \\ 1Department of Pathology, Seoul National University Bundang Hospital, Seongnam; \\ 2Department of Pathology, Seoul National University College of Medicine, Seoul, Korea
}

Programmed cell death protein 1/programmed death-ligand 1 (PD-1/PD-L1) inhibition has revolutionized the treatment paradigm of urothelial carcinoma (UC). Several PD-L1 assays are conducted to formulate appropriate treatment decisions for PD-1/PD-L1 target therapy in UC. However, each assay has its own specific requirement of antibody clones, staining platforms, scoring algorithms, and cutoffs for the determination of PD-L1 status. These prove to be challenging constraints to pathology laboratories and pathologists. Thus, the present article comprehensively demonstrates the scoring algorithm used and differences observed in each assay $(22 \mathrm{C} 3$, SP142, and SP263). Interestingly, the SP142 score algorithm considers only immune cells and not tumor cells (TCs). It remains controversial whether SP142 expressed only in TCs truly accounts for a negative PD-L1 case. Moreover, the scoring algorithm of each assay is complex and divergent, which can result in inter-observer heterogeneity. In this regard, the development of artificial intelligence for providing assistance to pathologists in obtaining more accurate and objective results has been actively researched. To facilitate efficiency of PD-L1 testing, several previous studies attempted to integrate and harmonize each assay in UC. The performance comparison of the various PD-L1 assays demonstrated in previous studies was encouraging, the exceptional concordance rate reported between 22C3 and SP263. Although these two assays may be used interchangeably, a clinically validated algorithm for each agent must be applied.

Key Words: Urothelial carcinoma; Programmed cell death-ligand 1; 22C3; SP142; SP263; Immunotherapy

Received: January 26, 2021 Accepted: February 22, 2021

Corresponding Author: Gheeyoung Choe, MD, PhD, Department of Pathology, Seoul National University Bundang Hospital, 82 Gumi-ro 173beon-gil, Bundang-gu, Seongnam 13620, Korea

Tel: +82-31-787-7711, Fax: +82-31-787-4012, E-mail: gychoe@snu.ac.kr

The U.S. Food and Drug Administration (FDA) has approved the use of programmed cell death protein 1/programmed deathligand 1 (PD-1/PD-L1) inhibitors (pembrolizumab, nivolumab, atezolizumab, and durvalumab) in the treatment of various cancers. PD-1/PD-L1 target therapies are no longer limited to tumor subtypes or origins. The interesting emerging concept of 'PDLoma' refers to tumors that respond to PD-1/PD-L1 target therapy [1]. Urothelial carcinoma (UC) is one of the most significant PD-Lomas. Particularly, pembrolizumab and atezolizumab are indicated as first-line treatments in patients with locally advanced or metastatic UC who are not eligible for cisplatin-containing chemotherapy and whose tumors are PD-L1 immunohistochemistry (IHC)-positive. PD-L1 IHC is a pivotal diagnostic technique used for determining the necessity of PD-1/PD-L1 target therapy. All agents are FDA-approved, used in conjunction with one of the PD-L1 assays available (22C3, 28-8, SP263, and SP142)_each of which involves different antibody clones, autostainers, scoring algorithms, and cutoffs $[2,3]$. This complexity implicated in the usage of PD-L1 assays has raised questions on their comparability and interchangeability. Although previous studies have attempted to integrate and harmonize the PD-L1 assays in non-small cell lung cancer (NSCLC), discordant PD-L1 expression was observed across the results of various assays [4-6]. Similarly, in UC, although a good correlation between each assay was observed, none exhibited a perfect agreement [3,7-9].

Diagnostic assays can be essential for the use of therapeutics (companion diagnostics) or may inform on improving the benefit without restricting drug access (complementary diagnostics) [10]. Notably, 22C3 and SP142 were companion diagnostics in the first-line use of pembrolizumab and atezolizumab, respectively $[11,12]$. However, 28-8 and SP263 have not been used as com- 
panion diagnostics for nivolumab and durvalumab in advanced UC patients $[13,14]$. Thus, the interpretation of $22 \mathrm{C} 3$ and SP142 may be crucial in practice and should be carefully assessed by pathologists. PD-L1 expression in immune cells (ICs) is comparatively as significant as that in tumor cells (TCs) in UC. The correlation between IC PD-L1 expression and treatment response has been demonstrated in all clinical studies conducted on UC, except in the case of nivolumab/28-8 [14].

In this review, we have discussed the scoring algorithm and differences in each PD-L1 assay in detail (Table 1) and assessed the current issues posed by PD-L1 testing in UC. Since the 28-8 assay is rarely used in most countries, including South Korea, 22C3, SP142, and SP263 in UC were evaluated.

\section{COMPARISON OF PD-L1 ASSAY INTERPRETATION IN UROTHELIAL CARCINOMA}

\section{Agilent 22C3}

According to the 22C3 (pharmDx) interpretation manual, PD-L1 expression was determined by using the combined positive score (CPS) in UC, which is the number of PD-L1-stained cells (TC + IC) divided by the total number of viable TCs, and multiplied by 100 (Table 1, Fig. 1) [15]. The result of the calculation can exceed 100; however, the maximum score is defined as CPS 100. The CPS is defined accordingly:

\section{CPS $=$ Number of PD-L1 staining cells $($ TCs + ICs $) /$ Total number of viable TCs $\times 100$}

ICs include lymphocytes and macrophages, but do not include plasma cells, neutrophils, and eosinophils. TCs with partial or complete linear membrane staining (at any intensity) were considered 'TC-positive.' ICs within the tumor nests and/or the immediately adjacent supporting stroma with convincing membrane and/or cytoplasmic staining (at any intensity) were considered 'IC-positive.' PD-L1 expression and CPS are suggested to be evaluated at higher magnification $(20 \times)$. Infiltrating UC, highgrade papillary UC, carcinoma in situ, and metastatic UC are included under CPS, whereas low-grade papillary UC and tumor necrotic area should be excluded. Finally, $22 \mathrm{C} 3$ is defined as positive if CPS $\geq 10$ in UC.

\section{Ventana SP142}

SP142 is scored as the proportion of tumor area that is occupied by PD-L1-expressing ICs at any intensity (Table 1, Fig. 1). Unlike 22C3, SP142 measures the area occupied instead of the number of stained cells. ICs include lymphocytes, macrophages, dendritic cells, and granulocytes, wherein stained ICs can be found as aggregates in intratumoral or contiguous peritumoral stroma, or as single cell spread among TCs. Tumor area for PDL1 scoring is defined as the area occupied by viable TCs and their associated intratumoral and contiguous peritumoral stroma. In papillary UC, the stroma in fibrovascular cores is considered intratumoral stroma. Tumor necrosis should be excluded for scoring. SP142 staining at any intensity of tumor-infiltrating ICs covering $\geq 5 \%$ of the tumor area is considered positive.

\section{Ventana SP263}

According to the manufacturer's manual, SP263 status is determined by the percentage of TCs with any membrane staining, or by the percentage of tumor-associated ICs with staining at any intensity (Table 1, Fig. 1). Similar to SP142, SP263 expresses the area proportionate to the tumor area measured. The percentage of tumor area occupied by any tumor-associated ICs (Immune Cells Present, ICP) is used to determine IC expression, which is defined as the percentage area of ICP exhibiting PD-L1-positive IC staining. SP263 status is considered positive if any of the following criteria are met:

$-\geq 25 \%$ of the TCs exhibit membrane staining; or,

Table 1. Comparison of PD-L1 assays for UC and difference in scoring algorithm

\begin{tabular}{|c|c|c|c|}
\hline & $22 \mathrm{C} 3$ & SP142 & SP263 \\
\hline Manufacturer & Agilent & Ventana & Ventana \\
\hline Drug & Pembrolizumab & Atezolizumab & Durvalumab \\
\hline Status & Companion diagnostic & Companion diagnostic & Complementary diagnostic \\
\hline Scoring algorithm & $\mathrm{CPS}=\# \mathrm{TC}+$ and $\# \mathrm{IC}+/$ Total $\# \mathrm{TC} \times 100 \geq 10$ & $\mathrm{IC}+$ tumor area $\geq 5 \%$ & $\begin{array}{l}\mathrm{TC}+/ \text { tumor area or } \geq 25 \% \\
\mathrm{ICP}>1 \%: \mathrm{IC}+/ \mathrm{ICP} \geq 25 \% \text { or } \\
\mathrm{ICP}=1 \%: \mathrm{IC}+/ \mathrm{ICP}=100 \%\end{array}$ \\
\hline Algorithm based on & Positive cell number & Positive cell area & Positive cell area \\
\hline Cell type & Tumor cells, lymphocytes, and macrophages & $\begin{array}{l}\text { Lymphocytes, macrophages, dendritic } \\
\text { cells and granulocytes }\end{array}$ & $\begin{array}{l}\text { Tumor cells, lymphocytes, macrophages, } \\
\text { histiocytes, plasma cells, and neutrophils }\end{array}$ \\
\hline
\end{tabular}

PD-L1, programmed cell death-ligand 1; UC, urothelial carcinoma; CPS, combined positive score; TC, tumor cell; IC, immune cell; ICP, immune cells present. 


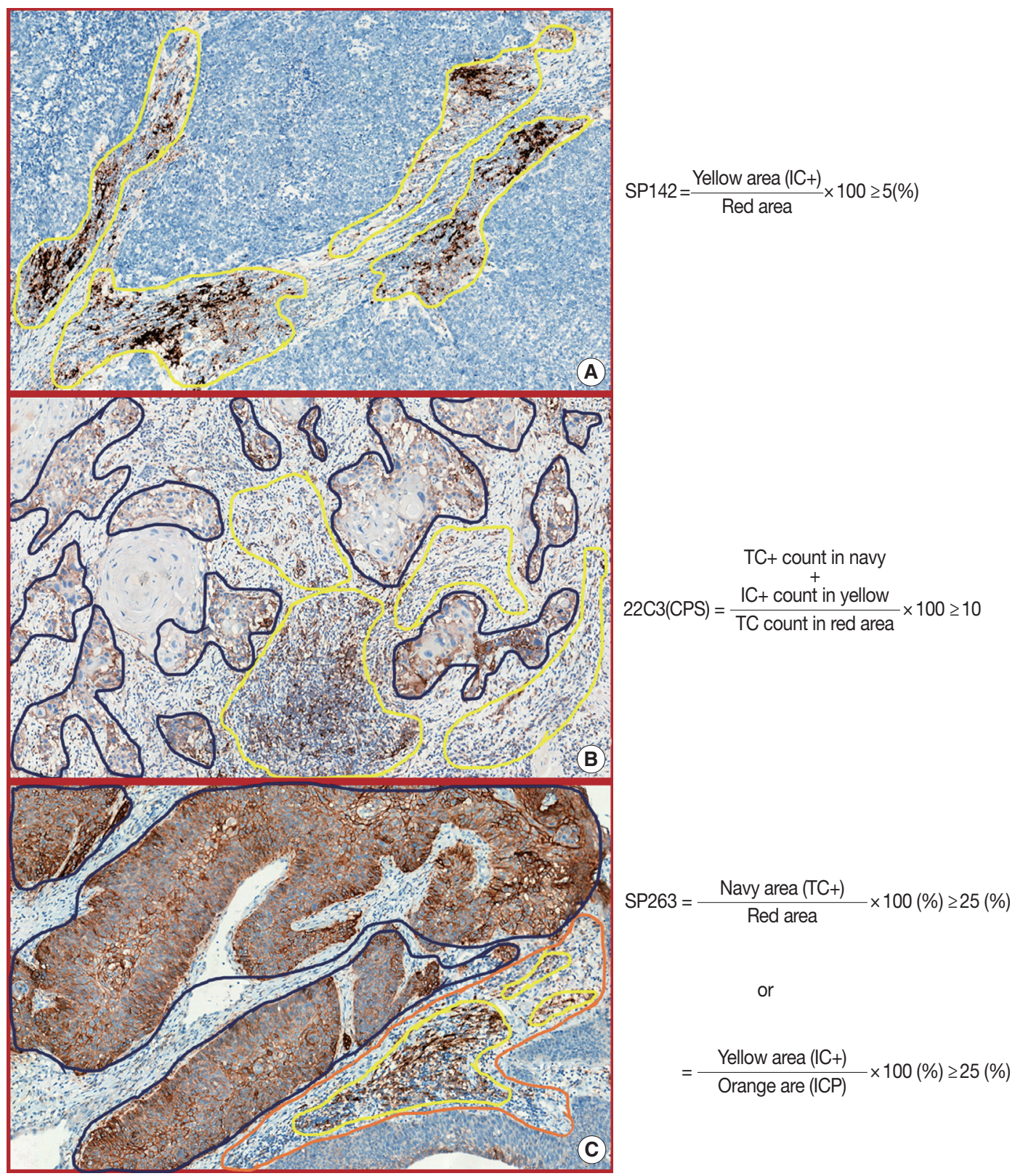

Fig. 1. Representative pictures for the comparison of programmed death-ligand 1 (PD-L1) assays and differences in scoring algorithm of urothelial carcinoma (UC). (A) Scoring algorithm of SP142 is based on the proportion of tumor area that is occupied by PD-L1-expressed immune cells (ICs) at any intensity. (B) Scoring algorithm of 22C3 is determined by using the combined positive score (CPS) in UC, which is the number of PD-L1-stained cells (tumor cell [TC] plus IC) divided by the total number of viable TCs, multiplied by 100. (C) SP263 status is determined by the percentage of TCs obtained by performing any membrane staining or by the percentage of tumor-associated ICs obtained by staining at any intensity. SP263-expressed TC area proportion of the tumor area is determined. Also, the percentage of tumor area occupied by any tumor-associated IC (Immune Cells Present, ICP) is used to determine IC expression, and IC positivity is defined as the percentage of PD-L1-positive IC area in ICP.

$$
\begin{aligned}
& -\mathrm{ICP}>1 \% \text { and } \mathrm{IC}+\geq 25 \% \text {; or, } \\
& -\mathrm{ICP}=1 \% \text { and } \mathrm{IC}+=100 \% \text {. }
\end{aligned}
$$

The manufacturer's manual suggests that an expression level greater than or equal to $25 \%$ of the TCs or ICs should be considered significant. Membrane staining of TCs can exhibit a partial or complete circumferential pattern. TC cytoplasmic staining is disregarded when determining PD-L1 expression. The percentage of tumor-associated IC with staining is evaluated in addition to TC staining. Interestingly, IC scoring includes lymphocytes, macrophages, histiocytes, plasma cells, and neutrophils. IC staining is 
assessed by initially reviewing the entire tumor area and by determining the ICP. Subsequently, the percentage of PD-L1 expressing ICs within the ICP is visually estimated (IC+). Additionally, in cases where the percentage of ICP in the tumor area is $1 \%$, it is considered positive only when $100 \%$ of the ICs are stained.

\section{INTERCHANGEABILITY OF PD-L1 ASSAYS IN UROTHELIAL CARCINOMA}

The use of different expensive autostainers and various assays is neither economical nor reasonable for pathology laboratories. Interchangeability of different assays may enable the usage of only one standardized PD-L1 assay in laboratories. In NSCLC, Adam et al. [16] showed that 28-8, 22C3, and SP263 assays demonstrated close analytical performance for TC staining across seven centers. However, a significant discrepancy was observed between SP142 and the other three assays for TC staining, whereas IC staining results were similar [17-19]. Moreover, the SP142 assay was an outlier that detected markedly less PD-L1 expression in both TCs and ICs [5].

Rijnders et al. [3] have suggested that agreement in the PDL1 status in UC between 22C3, 28-8, SP142, and SP263 is substantial (80\%-90\%), implying that these assays may be interchangeable in clinical practice. Moreover, a collaborative study conducted by the Russian Society of Clinical Oncology and the Russian Society of Pathology found that a patient with UC classified as negative by one of the three tests (22C3, SP142, and SP263) using the corresponding cutoff rule was highly likely (91\%-100\%) to be classified as negative based on the results of any other test performed, therefore avoiding the need for repeated testing [8]. Furthermore, Zajac et al. [9] reported a high level of analytical concordance among the SP263, 22C3, and 28-8 assays for TC and IC staining; however, such a level of concordance was not observed for SP142. Additionally, Hodgson et al. [7] demonstrated that SP142 TC staining intensity was lower in UC and hypopharyngeal squamous cell carcinoma samples, although there existed adequate analytic comparability between $22 \mathrm{C} 3$ and SP263. Another recent study has highlighted greater differences in the assays used for the analysis of PD-L1-stained TCs, particularly between SP142 and other assays [20]. These analytical findings were consistent with other studies conducted using NSCLC samples, which suggested that SP142 did not exhibit sufficient concordance with the other three assays. While 22C3 and SP263 have a high concordance rate and can be used interchangeably, clinical validation for each immune-therapy remains a necessity [9]. The interchangeability of PD-L1 assay must be considered carefully so as to ensure that no patient is devoid of treatment opportunity.

\section{INTER-OBSERVER HETEROGENEITY}

Inter-observer variability may lead to the obtainment of discordant results for PD-L1, which can consequentially impact therapy decisions. A recent study demonstrated that inter-observer agreement for each assay is moderate to high for IC staining (0.532-0.729) as well as TC staining (0.609-0.883) based on intra-class correlation coefficient obtained for UC [20]. However, $22 \mathrm{C} 3$ and 28-8 exhibited low inter-observer correlation in IC staining, while SP142 showed low inter-observer correlation in TC staining [20]. Downes et al. [21] suggested that excellent interobserver agreement could be found using SP263 and 22C3, whereas PD-L1 scoring using SP142 was associated with a higher level of subjectivity in head and neck squamous cell carcinoma, breast carcinoma, and UC. The study of inter-observer heterogeneity of PD-L1 assays has also been well conducted using NSCLC samples. According to Cooper et al. [22], 10 pathologists reported good reproducibility at both $1 \%$ cutoffs of $22 \mathrm{C} 3$, whereas agreement was slightly lower for the 50\% cutoff. Moreover, the Cardiopulmonary Pathology Study Group of the Korean Society of Pathologists investigated the inter-observer heterogeneity of PD-L1 staining with 22C3 using NSCLC samples [23]. Inter-observer reproducibility for the $1 \%$ cutoff was found to be relatively lower than the $50 \%$ cutoff, in contrast to the results reported by Cooper et al. [22]. Similarly, Rimm et al. [5] indicated that 13 pathologists reported excellent concordance when scoring TCs stained with any antibody (22C3, 28-8, SP142, and E1L3N) but reported poor concordance when scoring ICs stained with any antibody using NSCLC samples [16]. Although differing results were reported in previous studies of both UC and NSCLC, inter-observer heterogeneity of PD-L1 seems to occur.

\section{CORRELATION BETWEEN PD-L1 EXPRESSION AND HISTOLOGIC SUBTYPES}

UC is among the most histologically diverse cancers. A previous study found that infiltrating UC exhibits significantly higher $\mathrm{T}$ cell infiltration and PD-L1 expression than non-invasive papillary UC and UC in situ [24]. In addition to the conventional morphology observed, UC can contain elements of squamous differentiation, glandular differentiation, nested, plasmacytoid, sarcomatoid, and/or rarer variants. UC patients with histologic variants account for up to one-third of advanced cases. Li et al. 
[24] found that PD-L1 was expressed in a significant percentage of histologic variant of UC cases (cutoff 1\% TC, 37\% to 54\%; cutoff $5 \%$ TC, $23 \%$ to 37\%), while the highest PD-L1 expression was observed in patients with UC exhibiting squamous differentiation [25]. These results suggest that patients with histologic variants of UC may benefit more from anti-PD-1/PD-L1 therapy.

\section{DISCUSSION}

PD-1/PD-L1 target therapy has garnered considerable attention as a potential treatment strategy for patients with advanced UC. These agents are approved by the U.S. Food and Drug Administration for the treatment of patients with locally advanced or metastatic UC, with disease progression during or following platinum-containing chemotherapy, or disease progression within 12 months of neoadjuvant or adjuvant treatment with platinumcontaining chemotherapy. Moreover, pembrolizumab and atezolizumab have received approval for first-line treatment of locally advanced or metastatic UC in patients ineligible for cisplatincontaining chemotherapy. PD-L1 expression levels in UC can thus effectively aid physicians in identifying patients who are more likely to benefit from anti-PD-1/PD-L1 therapy.

However, every single agent is tested in conjunction with a specific PD-L1 assay, which must be performed on a specific staining platform. Moreover, the PD-L1 scoring algorithm is heterogeneous and unique for each assay. These aspects have encouraged pathologists to consider assay interchangeability. Several studies have attempted to harmonize PD-L1 assays conducted for NSCLC and UC samples. However, SP142 was an outlier that detected markedly less PD-L1 expression in TC (Fig. 2A-C) [5-7,9,18,20]. In contrast, relatively high concordance was observed between SP263 and 22C3. As each assay is performed using different immunogens, and thus a unique epitope, different PD-L1 conformations or isoforms may lead to the obtainment of heterogeneous results. Moreover, the location of the antibody-binding domain has been known to affect the staining pattern, resulting in increased variability [26]. This discordance in the results suggests that the prospects for interchangeability of the assays is not optimistic. Although the discordance rate between SP263 and 22C3 is low, there is no scientific evidence to prove that two assays can be used interchangeably. It is of utmost priority that all patients should receive proper treatment, without any exceptions arising due to assay discordance.

The scoring algorithm for each PD-L1 assay in UC is described in Table 1 and Fig. 1. There are several common practical diffi- culties in analyses performed using PD-L1 assays. First, it is difficult to distinguish between TC and IC positivity. Representatively, distinguishing between TCs and macrophages proves burdensome when PD-L1 is stained, because macrophages are of comparative size to the TCs (Fig. 2H). Reviewing the hematoxylin and eosin-stained slides can be helpful in this respect. $22 \mathrm{C} 3$ should be used to count both ICs and TCs, which may not be problematic; however, SP142 should be excluded PDL1-positive TCs. Moreover, ICs commonly include lymphocytes and macrophages. However, the ICs of SP263 additionally include plasma cells and neutrophils. Although this can also prove to be beneficial if we compare the hematoxylin and eosinstained slides with PD-L1-stained slides, it remains uncertain whether PD-L1-positive neutrophils, plasma cells, and lymphocytes can be distinguished effectively (Fig. 2I). Lastly, the lamina propria at the base of the papillary lesion may contain lymphoid aggregates that show PD-L1 positivity, whereas only the lamina propria contiguous to the base of the tumor is considered part of the tumor area. Moreover, in fragmented tissue samples, including transurethral resection or biopsies - where distinction of intra- or peritumoral stroma cannot be ascertained—only stroma that is contiguous to individual tumor nests is included in the tumor area definition (Fig. 2G). However, the meaning of 'contiguous' is ambiguous and subjective.

The 22C3 scoring system uses the CPS algorithm. Theoretically, regardless of whether the number of TCs is large or small, $22 \mathrm{C} 3$ should be used to count all TCs present in the tumor area for the denominator. Additionally, all PD-L1-positive TCs and ICs should be counted in the tumor area for obtaining values for the numerator. This scoring algorithm is labor-intensive and an accurate calculation is practically impossible for the whole tumor area. Thus, the 22C3 manufacturer (Agilent) suggests that a partial portion of the tumor can be selected and scored. However, these results may be inconsistent due to intratumoral heterogeneity of $22 \mathrm{C} 3$ expression.

A comparatively higher positive cutoff ( $\geq 25 \%$ of TC) is required for SP263. If the test results do not meet the TC cutoff, the IC cutoff ( $\geq 25 \%$ of IC) can be evaluated subsequently. Unlike SP142, the total tumor area is not evaluated for IC scoring. IC positivity is only evaluated in the ICP. Therefore, SP263 results may easily meet the cutoff ( $\geq 25 \%$ of IC) because ICP is relatively smaller than the total tumor area values used as a denominator. However, the evaluation of complex geographic ICP may pose challenges. For accurate evaluation, one must physically draw and cut out the ICP—which lies beyond the confines of plausibility. Hence, advances in artificial intelligence and digital pathology are nec- 

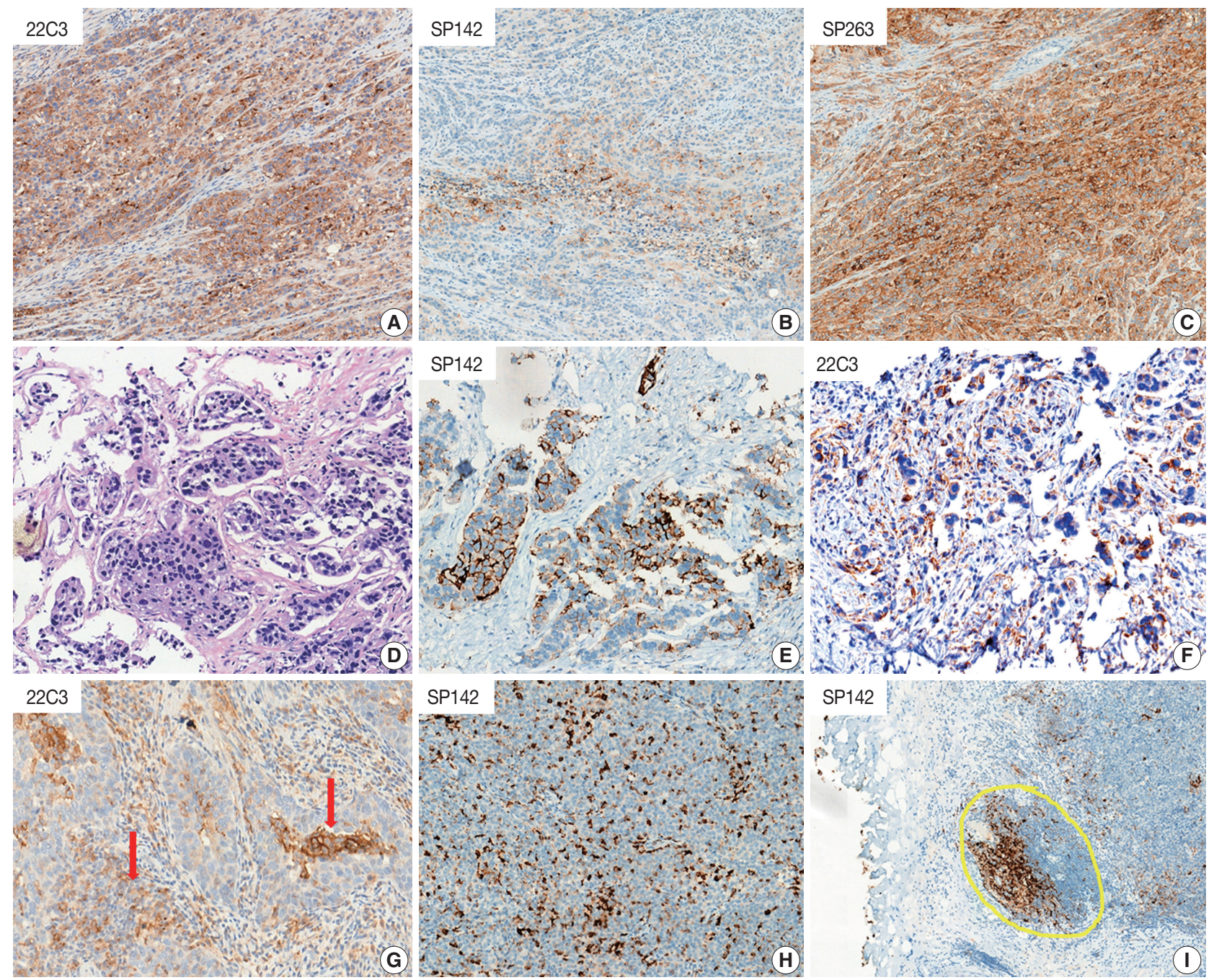

Fig. 2. (A-C) Representative pictures of heterogeneity observed in programmed death-ligand 1 (PD-L1) assay results (22C3, SP142, and SP263). SP142 was an outlier that detected markedly less PD-L1 expression in tumor cells (TCs). (D-F) Biopsy sample of metastatic urothelial carcinoma with PD-L1 expression observed only in TCs. Although SP142 was expressed in TCs, the result obtained was negative. However, the result for 22C3 was positive. This patient may be administered with pembrolizumab, but not with atezolizumab, as first-line therapy. (G) It is difficult to distinguish between the various subtypes of immune cells. If the regions indicated by the red arrows are plasma cells, they should be excluded from the 22C3 score. $(H)$ Investigation may be necessary to ascertain whether the SP142-stained cells are TCs or ICs. This picture shows SP142 expression on intra-tumoral macrophages. (I) In fragmented tissue samples, including transurethral resection or biopsy samples, where the distinction between intratumoral or peritumoral stroma cannot be clearly observed. The yellow area contiguous to the base of the tumor is considered part of the tumor area.

essary for the precise assessment of SP263 expression. At present, SP263 is not clinically used as a companion diagnostic for durvalumab in UC.

SP142 evaluation for the determination of the IC-positive area and score seems relatively simpler than performing other assays. However, exclusion of SP142 TC positivity information from scoring may not be an appropriate approach. We analyzed three UC biopsy cases (unpublished data), which showed only strong PD-L1 positivity in TCs and not in ICs, with negative SP142 results (Fig. 2D-F). Although all the UC cases studied herein were SP142-negative, these patients demonstrated a complete response to atezolizumab. Whilst these results were obtained for only a limited number of cases, it should nonetheless be considered whether the exclusion of positive TCs from the scoring algorithm is a reasonable methodology. The ability of SP142 to detect TC expression is low, which may lead to the generation of statistical bias in clinical trials of atezolizumab in UC. This is probably main reason that TC were not included in the scoring algorithm of the SP142. Re-evaluation of SP142 TC expression may thus be necessary in a novel clinical study to assess anti-PD-1/ 
PD-L1 therapy in UC. Furthermore, in cases where SP142 expression is observed only in TCs, it is recommended to adopt the $22 \mathrm{C} 3$ test to determine the applicability of pembrolizumab as first-line treatment.

These complex score algorithms and intratumoral heterogeneity of PD-L1 expression can result in inter-observer heterogeneity, particularly in scoring the SP142 of UC [20,21]. It is not difficult to score definite PD-L1 positive or negative cases; however, inter-observer heterogeneity must be observed in cases with approximate cutoff scores (e.g., 22C3 CPS, 5\%-15\%; SP142, $3 \%-10 \%$, and so on). Providing training to the pathologist in these aspects as well as in the use of artificial intelligence may be a possible method for reduction of the inter-observer PD-L1 discordance.

Several studies have suggested the prognostic significance of PD-L1 expression in various malignancies; PD-L1-expressing tumors tend to exhibit poor prognosis [27]. Previous metaanalyses have demonstrated that PD-L1 expression is correlated with worse prognosis and advanced clinicopathological features in UC [28,29]. Moreover, Kawahara et al. indicated that UC with high-grade features exhibited higher PD-L1 expression [30]. A recent study has reported that the increased expression of PDL1 is correlated with histologic variants of UC, including squamous, glandular, plasmacytoid, and sarcomatoid differentiation [25]. Histologic variants of UC constitute a high-grade feature that tends to be associated with PD-L1 expression. In this regard, PD-1/PD-L1 target therapy may be more effective for the treatment of histologic variants of UC. Although PD-L1 positivity is an important predictor of anti-PD-1/PD-L1 treatment response, PD-L1 expression status alone is insufficient to determine prognosis in any cancer subtype.

\section{CONCLUSION}

We reviewed the prospect and existing limitations of PD-L1 assays performed using samples from patients with UC. Discordance of PD-L1 positivity was observed, depending on the results of each assay. Notably, the inter-assay and inter-observer discordance were primarily observed in scoring SP142. Thus far, there exists no scientific evidence for the interchangeability of PD-L1 assays. If atezolizumab cannot be used as first-line therapy due to SP142 positivity observed only in TCs, and not in ICs, we recommend adopting the use of $22 \mathrm{C} 3$ in conjunction with pembrolizumab. The complex scoring algorithm of each assay is challenging for pathologists and also results in inter-observer heterogeneity. Providing suitable training to pathologists may be the only ap- proach to overcome these challenges. Moreover, in the future, digital pathology and artificial intelligence may assist PD-L1 evaluation with greater accuracy.

\section{Ethics Statement}

Not applicable.

\section{Availability of Data and Material}

Data sharing not applicable to this article as no datasets were generated or analyzed during the study.

\section{Code Availability}

Not applicable.

\section{ORCID}

Kyu Sang Lee https://orcid.org/0000-0003-2801-9072 Gheeyoung Choe https://orcid.org/0000-0001-6547-5603

\section{Author Contributions}

Conceptualization: KSL, GC. Data curation: KSL. Formal analysis: KSL. Investigation: KSL. Methodology: KSL. Supervision: GC. Writing_original draft: KSL. Writing—review \& editing: KSL, GC. Approval of final manuscript: all authors.

\section{Conflicts of Interest}

The authors declare that they have no potential conflicts of interest.

\section{Funding Statement}

No funding to declare.

\section{References}

1. Hirsch L, Zitvogel L, Eggermont A, Marabelle A. PD-Loma: a cancer entity with a shared sensitivity to the PD-1/PD-L1 pathway blockade. Br J Cancer 2019; 120: 3-5.

2. Kerr KM, Tsao MS, Nicholson AG, et al. Programmed death-ligand 1 immunohistochemistry in lung cancer: in what state is this art? J Thorac Oncol 2015; 10: 985-9.

3. Rijnders M, van der Veldt AA, Zuiverloon TC, et al. PD-L1 antibody comparison in urothelial carcinoma. Eur Urol 2019; 75: 538-40.

4. Ratcliffe MJ, Sharpe A, Midha A, et al. Agreement between programmed cell death ligand-1 diagnostic assays across multiple protein expression cutoffs in non-small cell lung cancer. Clin Cancer Res 2017; 23: 3585-91.

5. Rimm DL, Han G, Taube JM, et al. A prospective, multi-institutional, pathologist-based assessment of 4 immunohistochemistry assays for PD-L1 expression in non-small cell lung cancer. JAMA Oncol 2017; 3: 1051-8.

6. Scheel AH, Dietel M, Heukamp LC, et al. Harmonized PD-L1 immunohistochemistry for pulmonary squamous-cell and adenocarcinomas. Mod Pathol 2016; 29: 1165-72.

7. Hodgson A, Slodkowska E, Jungbluth A, et al. PD-L1 immunohistochemistry assay concordance in urothelial carcinoma of the bladder and hypopharyngeal squamous cell carcinoma. Am J Surg Pathol 2018; 42: 1059-66.

8. Zavalishina L, Tsimafeyeu I, Povilaitite P, et al. RUSSCO-RSP comparative study of immunohistochemistry diagnostic assays for PDL1 expression in urothelial bladder cancer. Virchows Arch 2018; 
473: 719-24.

9. Zajac M, Scott M, Ratcliffe M, et al. Concordance among four commercially available, validated programmed cell death ligand-1 assays in urothelial carcinoma. Diagn Pathol 2019; 14: 99.

10. Scheerens H, Malong A, Bassett K, et al. Current status of companion and complementary diagnostics: strategic considerations for development and launch. Clin Transl Sci 2017; 10: 84-92.

11. Rosenberg JE, Hoffman-Censits J, Powles T, et al. Atezolizumab in patients with locally advanced and metastatic urothelial carcinoma who have progressed following treatment with platinum-based chemotherapy: a single-arm, multicentre, phase 2 trial. Lancet 2016; 387: 1909-20.

12. Balar AV, Castellano D, O'Donnell PH, et al. First-line pembrolizumab in cisplatin-ineligible patients with locally advanced and unresectable or metastatic urothelial cancer (KEYNOTE-052): a multicentre, single-arm, phase 2 study. Lancet Oncol 2017; 18: 1483-92.

13. Powles T, O'Donnell PH, Massard C, et al. Efficacy and safety of durvalumab in locally advanced or metastatic urothelial carcinoma: updated results from a phase 1/2 open-label study. JAMA Oncol 2017; 3: e172411.

14. Sharma P, Retz M, Siefker-Radtke A, et al. Nivolumab in metastatic urothelial carcinoma after platinum therapy (CheckMate 275): a multicentre, single-arm, phase 2 trial. Lancet Oncol 2017; 18: 31222.

15. Kulangara K, Zhang N, Corigliano E, et al. Clinical utility of the combined positive score for programmed death ligand-1 expression and the approval of pembrolizumab for treatment of gastric cancer. Arch Pathol Lab Med 2019; 143: 330-7.

16. Adam J, Le Stang N, Rouquette I, et al. Multicenter harmonization study for PD-L1 IHC testing in non-small-cell lung cancer. Ann Oncol 2018; 29: 953-8.

17. Torlakovic E, Lim HJ, Adam J, et al. "Interchangeability” of PD-L1 immunohistochemistry assays: a meta-analysis of diagnostic accuracy. Mod Pathol 2020; 33: 4-17.

18. Hirsch FR, McElhinny A, Stanforth D, et al. PD-L1 immunohistochemistry assays for lung cancer: results from phase 1 of the Blueprint PD-L1 IHC assay comparison project. J Thorac Oncol 2017; 12: 208-22.

19. Buttner R, Gosney JR, Skov BG, et al. Programmed death-ligand 1 immunohistochemistry testing: a review of analytical assays and clinical implementation in non-small-cell lung cancer. J Clin Oncol
2017; 35: 3867-76

20. Schwamborn K, Ammann JU, Knuchel R, et al. Multicentric analytical comparability study of programmed death-ligand 1 expression on tumor-infiltrating immune cells and tumor cells in urothelial bladder cancer using four clinically developed immunohistochemistry assays. Virchows Arch 2019; 475: 599-608.

21. Downes MR, Slodkowska E, Katabi N, Jungbluth AA, Xu B. Interand intraobserver agreement of programmed death ligand 1 scoring in head and neck squamous cell carcinoma, urothelial carcinoma and breast carcinoma. Histopathology 2020; 76: 191-200.

22. Cooper WA, Russell PA, Cherian M, et al. Intra- and interobserver reproducibility assessment of PD-L1 biomarker in non-small cell lung cancer. Clin Cancer Res 2017; 23: 4569-77.

23. Chang S, Park HK, Choi YL, Jang SJ. Interobserver reproducibility of PD-L1 biomarker in non-small cell lung cancer: a multi-institutional study by 27 pathologists. J Pathol Transl Med 2019; 53: 34753.

24. Li H, Zhang Q, Shuman L, et al. Evaluation of PD-L1 and other immune markers in bladder urothelial carcinoma stratified by histologic variants and molecular subtypes. Sci Rep 2020; 10: 1439.

25. Reis H, Serrette R, Posada J, et al. PD-L1 expression in urothelial carcinoma with predominant or pure variant histology: concordance among 3 commonly used and commercially available antibodies. Am J Surg Pathol 2019; 43: 920-7.

26. Mahoney KM, Sun H, Liao X, et al. PD-L1 antibodies to its cytoplasmic domain most clearly delineate cell membranes in immunohistochemical staining of tumor cells. Cancer Immunol Res 2015; 3 : 1308-15.

27. Wu P, Wu D, Li L, Chai Y, Huang J. PD-L1 and survival in solid tumors: a meta-analysis. PLoS One 2015; 10: e0131403.

28. Ding X, Chen Q, Yang Z, et al. Clinicopathological and prognostic value of PD-L1 in urothelial carcinoma: a meta-analysis. Cancer Manag Res 2019; 11: 4171-84.

29. Wen Y, Chen Y, Duan X, et al. The clinicopathological and prognostic value of PD-L1 in urothelial carcinoma: a meta-analysis. Clin Exp Med 2019; 19: 407-16.

30. Kawahara T, Ishiguro Y, Ohtake S, et al. PD-1 and PD-L1 are more highly expressed in high-grade bladder cancer than in low-grade cases: PD-L1 might function as a mediator of stage progression in bladder cancer. BMC Urol 2018; 18: 97. 